MATEC Web of Conferences 51, 02005 (2016)

DOI: $10.1051 /$ matecconf/20165102005

(C) Owned by the authors, published by EDP Sciences, 2016

\title{
Development and Analysis of Flexible Thin Actuator with a Built-in Fluid Pressure Source
}

\author{
Shinji Senzaki ${ }^{1}$,Tetsuya Akagi ${ }^{1, a}$, Shujiro Dohta ${ }^{1}$, and Yuto Fujiwara ${ }^{1}$ \\ ${ }^{1}$ Department of Intelligent Mechanical Engineering, Okayama University of Science \\ 1-1,Ridai-cho, Kita-ku, Okayama, 700-0005, Japan.
}

\begin{abstract}
A flexible thin actuator using gas-liquid phase-change of a low boiling point liquid that can generate large force was proposed and tested in the previous study. The tested actuator is an envelope-type actuator that is made of laminating plastic sheets, low boiling point liquid and a flexible heater. In this paper, the analytical model of the flexible thin actuator was proposed and tested. The system parameters of the actuator were also identified. As a result, it was confirmed that the proposed analytical model can predict the behaviour of the tested actuator.
\end{abstract}

\section{Introduction}

Due to the aging of Japanese society and the decreasing birth rate, an important problem to keep a quality of life (QOL) for the elderly has occurred[1]. Especially, the decrease in physical ability of the elderly will be concerned to increase in nursing care task. Therefore, the national budget for social welfare will be increased. In order to solve this problem, that is, to improve a quality of life (QOL) for the elderly and the disabled and to decrease the budget for nursing care, a simple rehabilitation device to keep their physical ability might be one of solutions. The actuator used in such a rehabilitation device requires lightweight, softness, small size and larger generated force that can support human body[2,3]. Therefore, many researchers are studying wearable actuators such as a McKibben artificial muscle[4,5]. The McKibben actuator can generate larger force that is more than $300 \mathrm{~N}$ by using the actuator whose diameter of $10 \mathrm{~mm}$ in the initial condition. However, the actuator can not work with long stroke. Maximum stroke is one fourth of the original length of the actuator. To get larger force by using other pneumatic actuator, larger sectional area of the actuator or higher pressure supplied to the actuator is needed. In ideal, a thin actuator that can generate larger force and larger displacement is required. Therefore, in our previous study[6], the envelope-type actuator driven by pneumatic pressure was proposed and tested as a flexible thin actuator. The actuator can be used as a rehabilitation device for shoulder by being sandwiched it in armpit. In addition, the flexible thin actuator using gas-liquid phase-change of low boiling point liquid was also proposed and tested[7]. It was also confirmed that the tested actuator could be driven silently. In the next step, it is necessary to develop the method to estimate the characteristics of the actuator to design it so as to get the suitable behaviour. In this paper, an analytical model of the tested actuator is proposed. The identified parameters and the comparison

\footnotetext{
${ }^{\mathrm{a}}$ Corresponding author : akagi@are.ous.ac.jp
} 
of the dynamic characteristics between the theoretical and the experimental results of the actuator will be also described.

\section{Flexible thin actuator}

In the pervious study, the thinner and lighter wearable actuator was proposed and developed[6]. Figure 1 shows the construction and the view of flexible thin actuator that is an envelope-type actuator. The actuator consists of four sheets of paper and plastic laminate films as shown in Fig.1. The left figure in Fig. 1 shows the shape of paper A and B. The middle figure shows the sectional structure of the actuator. The paper B works as a gusset in the actuator. The size of plastic laminate film is larger than the size of paper A and B. The plastic laminate films are bonded each other at the margin of the films with the exception of paper area. Then, the length of $L_{A}, L_{B}$ and $H$ in paper A and B are $90 \mathrm{~mm}$, $110 \mathrm{~mm}$ and $40 \mathrm{~mm}$, respectively. From Fig. 1 on the right, it can be seen that the thickness of the actuator was greatly deformed about 39 times from the initial thickness of $1.5 \mathrm{~mm}$ with no supplied pressure to maximum thickness of $58.4 \mathrm{~mm}$. By using typical laminate films and paper that can be easily got on the market as a stationery, the actuator can be quickly and easily fabricated. The time for production of the actuator is several minutes.

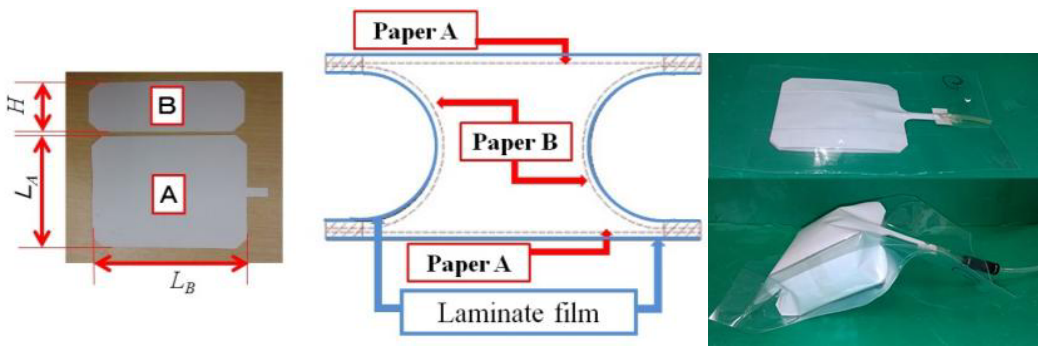

Figure 1. Contraction and view of the flexible thin actuator that is envelope-type actuator.

Figure 2 shows the relation between the expanded length (displacement) and the generated force of the actuator. From Fig.2, it can be seen that the maximum generated force of $657 \mathrm{~N}$ could be obtained in the case of supplied pressure of $100 \mathrm{kPa}$. From these results, the following experimental relation between the displacement and the generated force of the actuator can be obtained.

$$
F=A_{0} P\left(1-X / X_{M}\right)
$$

where, $F, A_{0}, P, X$ and $X_{M}$ mean the generated force, equivalent sectional area, supplied pressure, displacement and maximum displacement of the actuator, respectively. In Fig.2, the solid lines show calculated results using Eq.(1) with $A_{0}=0.8 L_{A} L_{B}=0.00792 \mathrm{~m}^{2}$ and $X_{M}=2.9 L_{C}=0.058 \mathrm{~mm}$. From Fig. 2 , it can be seen that the proposed experimental relation expressed by Eq.(1) about the generated force and displacement of the actuator can predict well the static characteristics of the tested actuator.

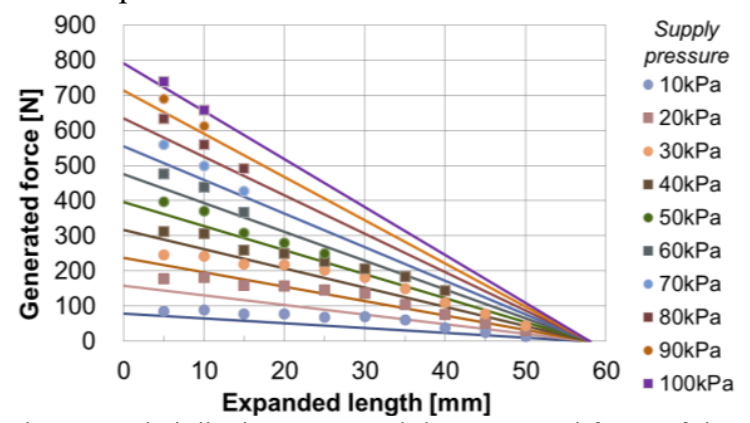

Figure 2. Relation between the expanded displacement and the generated force of the actuator. 


\section{Actuator with built-in fluid pressure source}

\subsection{Construction of thin actuator with built-in pressure source}

In order to develop the actuator driven by the gas-liquid phase change of the low boiling point liquid, the heat of evaporation and the boiling point of the liquid must be low from the view point of the energy consumption and the response for heater. In addition, from the view point of working liquid in a rehabilitation device, it must have safety, non-toxicity to the human body and harmless to the

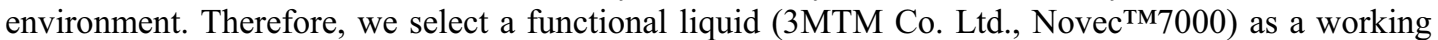
liquid. The liquid is used as a cleaning liquid of substrate in the electronic circuit. The boiling point is 34 degrees centigrade. The heat of evaporation is $142 \mathrm{~kJ} / \mathrm{kg}$. This value means about one sixteenth of that of that of case using the water $(\mathrm{kJ} / \mathrm{kg})$. The liquid is nonflammable and nonpoisonous fluid virtually. The high volume resistivity shows that the liquid can contact the electric heater directly during the high applied current.

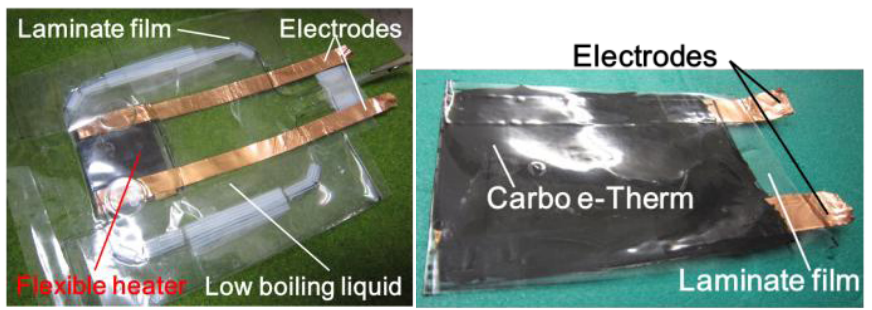

Figure 3. Construction of the flexible thin actuator with low boiling point liquid and flexible heater.

Figure 3 shows the construction of the flexible thin actuator using gas-liquid phase-change of low boiling point liquid and a flexible heater. The flexible heater consists of a copper tape as an electrode, a functional paint (Future Carbon GmbH Co. Ltd., Carbon e-Therm PUR-120) [8] and plastic laminate films. The size of the heater as shown in Fig. 3 on the left is $60 \times 65 \mathrm{~mm}$. The thickness of the heater is $0.5 \mathrm{~mm}$. The heater has flexibility. The flexible heater is installed into the envelope-type actuator with the low boiling point liquid. The liquid is used as a cleaning liquid for the electronic circuit board. The low boiling point liquid with volume of about $50 \mathrm{~cm}^{3}$ is also poured into the actuator. To apply the voltage from the outside of the actuator, two copper tapes were connected with the heater. The size of the actuator is $110 \mathrm{~mm}$ in length, $90 \mathrm{~mm}$ in width and $6 \mathrm{~mm}$ in thickness. The total mass of the actuator including the liquid is $80 \mathrm{~g}$.

\subsection{Fundamental characteristics}

Figure 4 shows the transient view of the movement of the tested actuator when the input voltage of $10 \mathrm{~V}$ was applied. Each figure shows the view of the actuator when 5, 10, 15 seconds from the beginning of starting to apply the voltage. It can be seen that the actuator reached at maximum displacement within 15 seconds. After stopping to apply the voltage, the actuator was contracted according to the decreasing the temperature of the liquid by natural cooling. It was confirmed that the actuator did not make any sound while being driven.
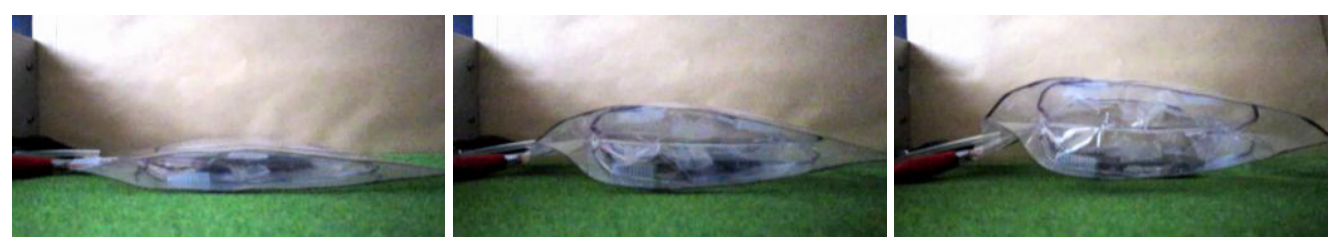

Figure 4. Transient view of movement of the tested actuator when the input voltage of $10 \mathrm{~V}$ is applied (Time:5s, $10 \mathrm{~s}, 15 \mathrm{~s})$. 
Figure 5 shows the experimental set up for measuring the displacement of the actuator and the transient response of the displacement of the tested actuator. In the experiment, the displacement of the actuator was measured by using the leaser displacement sensor (Optex FA Co. Ltd., CD33$120 \mathrm{NV})$. The input voltage of $8 \mathrm{~V}$ was applied for 20 seconds.
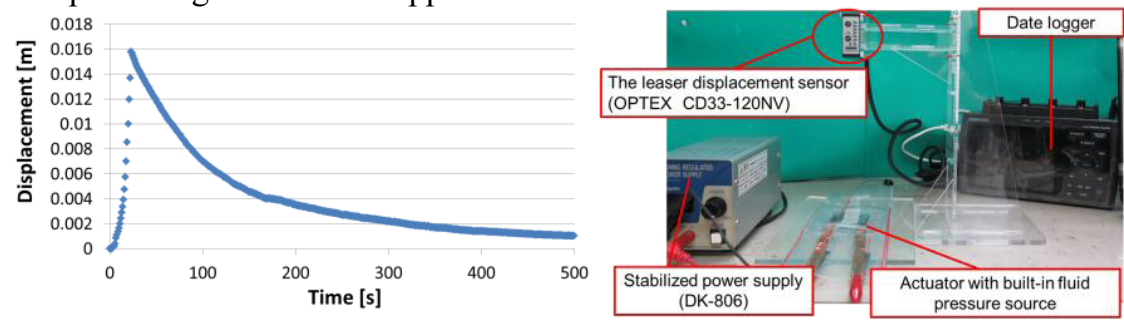

Figure 5. Transient response of the displacement of the actuator.

\section{Analytical model of the actuator}

In order to estimate the dynamics of the actuator, it is necessary to make an analytical model of gasliquid phase change of low boiling point liquid. Therefore, the following energy equilibrium between the inner thermal energy of the actuator and the energy given by the heater and the atmosphere is considered.

$$
m_{G}\left\{\left(T_{b}-T_{a}\right) \alpha+L\right\}=k_{w} \int W_{i n} d t-\int A_{h} U\left(T_{b}-T_{a}\right) d t
$$

where, $U, k_{w}, L, m_{G}, W_{i n}$ and $\alpha$ are the convective heat transfer coefficient, the efficiency of the heater, the latent heat of vaporization $(=142 \mathrm{~kJ} / \mathrm{kg})$, the mass of the gas, the heating value of the heater $(=16$ $\mathrm{W})$ and the specific heat $(=1300 \mathrm{~J} / \mathrm{kgK})$ of the low boiling point liquid, respectively. $T_{a}, T_{b}$ and $T_{0}$ mean the absolute temperature of the atmosphere $(=296 \mathrm{~K})$, the boiling point and the initial temperature of the liquid, respectively. Then, the left side term shows the energy required to make the liquid boiled. The right side terms express the energy from the heater and the air cooling, respectively. From Clausius-Clapeyron equation, the boiling point $T_{b}$ of the liquid in the actuator is changed according to the inner pressure $(=101.3 \mathrm{kPa})$ of the actuator $P_{i}$ and can be given by the following equation.

$$
T_{b}=\frac{T_{b a} L}{L-R T_{b a} \ln \left(P_{i} / P_{a}\right)}
$$

where, $P_{a}, R$ and $T_{b a}$ show the atmospheric pressure, the gas constant $(=287 \mathrm{~J} /(\mathrm{kg} \cdot \mathrm{K}))$ and the boiling point at the atmospheric pressure $(=307 \mathrm{~K})$,respectively. In Eq. (2), $A_{h}$ is the equivalent contacting area with the gas and given by

$$
A_{h}=L_{B}\left(2 L_{A}+4 L_{C}\right) \frac{V_{G a}}{V_{G a}+V_{L}}
$$

In Eq. (2), $U$ means convective heat transfer coefficient and is given by

$$
U=\frac{1}{\frac{1}{h_{g}}+\frac{t_{w}}{\lambda}+\frac{1}{h_{a}}}
$$

where, $t_{w}, h_{g}, h_{a}$ and $\lambda$ show the thickness, thermal conductivity of the actuator's material, gas and atmosphere solidification heat transfer coefficient, respectively.

Then, it is assumed that the pressure of the gas is equal to the atmospheric pressure by changing the inner volume of the actuator. The volume $V_{G a}$ of the gas that is vaporized from the liquid is given as follows.

$$
V_{G a}=\frac{m_{G}}{M_{L a}} M_{G a}
$$

where, $M_{L a}$ and $M_{G a}$ show the molar mass of the liquid $(=0.02006 \mathrm{~kg} / \mathrm{mol})$ and gas $\left(=22.4 \times 10^{-3}\right.$ $\mathrm{m}^{3} / \mathrm{mol}$ ) at the atmospheric pressure, respectively. 


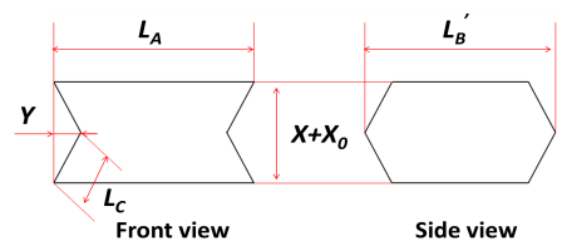

Figure 6. Geometric relationship of the actuator

Figure 6 shows the geometric relationship of the actuator. From Fig.6, the relation between the volume and the displacement of the actuator $x$ can be obtained as follows.

$$
\left(V_{G a}+V_{L}\right)=L_{A}\left\{\frac{\pi-4}{4}\left(x+x_{0}\right)^{2}+L_{B}^{\prime}\left(x+x_{0}\right)\right\}-Y L_{B}^{\prime}\left(x+x_{0}\right)
$$

where, $L_{B}^{\prime}$ and $Y$ are given by following equations.

$$
\begin{aligned}
& L_{B}^{\prime}=L_{B}+\frac{2-\pi}{4}\left(x+x_{0}\right) \\
& Y=\sqrt{L_{C}^{2}-\left(\frac{x+x_{0}}{2}\right)^{2}}
\end{aligned}
$$

The volume $V_{L}$ of the liquid is given by

$$
V_{L}=\frac{m_{\text {all }}-m_{G}}{\rho}
$$

where, $\rho, m_{G}$ and $m_{\text {all }}$ mean the density of the liquid, mass of gas and whole mass, respectively. By using Eqs. (2) to (10), the behavior of the actuator when a heat is applied can be calculated.

Table 1 shows the identified parameters of the actuator. Most parameters are measured and investigated from the specification. The parameter of $h_{g}$ and $k_{w}$ are identified so that the calculated result of the behavior of the actuator agrees with the experimental result.

Table 1. Identified parameters of the flexible thin actuator using the low boiling point liquid.

\begin{tabular}{|c|c|c|c|c|c|}
\hline Parameter & value & Parameter & value & Parameter & value \\
\hline$A_{h}\left(\mathrm{~m}^{2}\right)$ & variable & $t_{w}(\mathrm{~m})$ & 0.0003 & $\lambda(\mathrm{W} / \mathrm{mK})$ & 0.33 \\
$\alpha(\mathrm{J} / \mathrm{kgK})$ & 1300 & $m_{G}(\mathrm{~kg})$ & variable & $T a(\mathrm{~K})$ & 296 \\
$h_{a}\left(\mathrm{~W} / \mathrm{m}^{2} \mathrm{~K}\right)$ & 3,5 & $M_{G a}\left(\mathrm{~m}^{3} / \mathrm{mol}\right)$ & $22.4 * 10-3$ & $T b(\mathrm{~K})$ & variable \\
$h_{g}\left(\mathrm{~W} / \mathrm{m}^{2} \mathrm{~K}\right)$ & 6000 & $M_{L A}(\mathrm{~kg} / \mathrm{mol})$ & 0.02006 & $T b a(\mathrm{~K})$ & 307 \\
$k w$ & 0.55 & $P a(\mathrm{kPa})$ & 101.3 & $\operatorname{Vga}\left(\mathrm{m}^{3}\right)$ & variable \\
$L_{A}(\mathrm{~m})$ & 0.09 & $P i(\mathrm{kPa})$ & variable & $\operatorname{Win}(\mathrm{W})$ & 16 \\
$L_{B}(\mathrm{~m})$ & 0.11 & $R(\mathrm{~J} / \mathrm{mol} \cdot \mathrm{K})$ & 287 & $x(\mathrm{~m})$ & variable \\
$L_{C}(\mathrm{~m})$ & 0.02 & $L(\mathrm{~kJ} / \mathrm{kg})$ & 142 & $\rho\left(\mathrm{kg} / \mathrm{m}^{3}\right)$ & 1400 \\
\hline
\end{tabular}

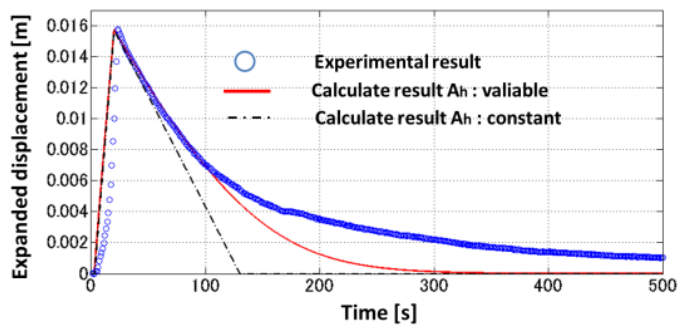

Figure 7. Transient response of the displacement of the actuator

Figure 7 shows the transient response of the measured and calculated displacement of the actuator by using the model and identified parameters. The experimental condition is same as the previous as shown in Fig.5. In Fig.7, symbols and lines show the experimental and calculated results, respectively. The solid line is the calculated result using the model in the case that the equivalent contacting area 
with the gas $A_{h}$ is variable. The broken line shows the calculated result in the case when $A_{h}$ is constant. It can be seen that the calculated result in the case that $A_{h}$ is variable agrees with the experimental results compared with the case when $A_{h}$ is constant. The difference between the experimental and calculated results was also found in the result after $100 \mathrm{~s}$ from the beginning. This is because the gas solidification heat transfer coefficient dose not change according to the condensation. Therefore, it is necessary to improve the analytical model as our further work. However, the calculation using the proposed model can mostly predict the behaviour of the actuator. It can be concluded that the proposed model and the identified parameters are valid to estimate the performance of the actuator theoretically.

\section{Conclusions}

This study that aims to develop and analyse the wearable thin actuator driven by low boiling point liquid for portable rehabilitation device can be summarized as follows. The analytical model of the actuator that includes the gas-liquid phase-change and the energy equilibrium between the inner thermal energy of the actuator and the given energy by the heater and the atmosphere was proposed and tested. The system parameters of the actuator were also identified. As a result, the calculated displacement of the actuator using the proposed model and the identified parameters can predict the behaviour of the tested actuator. It is confirmed that the proposed model is valid to estimate the dynamics of the tested actuator theoretically.

\section{Acknowledgements}

This work was partially supported by the Ministry of Education, Culture, Sports, Science and Technology of Japan through a QOL Innovative Research Program (2012-2016) and Grant-in-Aid for Scientific Research (C) (Subject No. 24560315).

\section{References}

1. Ministry of Internal Affairs and Communications, Statistics Bureau, Director-General for Policy Planning (Statistical Standards) \& Statistical Research and Training Institute, Statistical handbook of Japan 2013 [Online].

2. T. Akagi, S. Dohta, M. Ihara, Improvement of McKibben Artificial Muscle with Long Stroke Motion and Its Application, Journal of System Design and Dynamics, Vol.4, No.4, 538551(2010).

3. Y. Nagata ed., Soft Actuators -Forefront of Development-, NTS Ltd., 291- 335(2014)

4. H. Kobayashi, T. Shiban, Y. Ishida, Realization of all 7 motions for the upper limb by a muscle suit, Journal of Robotics and Mechatronics, Vol.16, No.5, 504-512(2004).

5. T. Noritsugu, M. Takaiwa, D. Sasaki, Development of Power Assist Wear Using Pneumatic Rubber Artificial Muscles, Journal of Robotics and Mechatronics, Vol.21, No.5, 607-613,(2009.)

6. Y. Tsuji, S. Dohta, T. Akagi S. Noguchi, Development of Envelope-type Pneumatic Thin Actuator, Proc. FLUCOME 2013, OS4-03-2pdf, 1-7,(2013)

7. $3 \mathrm{M}^{\mathrm{TM}}$ Novec ${ }^{\mathrm{TM}} 7000$ Engineered Fluid (Catalog) [Online]

8. T. Akagi, S. Dohta, S. Fujimoto, Y. Tsuji, Y. Fujiwara, Development of Flexible Thin Actuator Driven by Low Boiling Point Liquid, International Journal of Materials Science and Engineering Vol. 3, No. 1, March 55-58,(2015) 